\title{
HJELMSLEV QUADRILATERAL CENTRAL CONFIGURATIONS
}

\author{
MARTHA ALVAREZ-RAMÍREZ ${ }^{1}$ AND JAUME LLIBRE ${ }^{2}$
}

\begin{abstract}
A Hjelmslev quadrilateral is a quadrilateral with two right angles at opposite vertices. We classify all planar four-body central configurations where the four bodies are at the vertices of a Hjelmslev quadrilateral. We show that in the positive mass space $\left(m_{1}, m_{2}, m_{3}, m_{4}\right)$, taking the unit of mass equal to $m_{1}$, the set of Hjelmslev quadrilateral central configurations of the fourbody problem is an open arc. When the masses tend to the endpoints of this arc three of the masses of the Hjelmslev quadrilateral central configurations tend to an equilateral central configuration of the three-body problem, and the fourth remainder mass tends to zero.
\end{abstract}

\section{INTRODUCTION AND STATEMENT OF THE MAIN RESUltS}

The $n$-body problem, i.e. the description of the motion of $n$ particles of positive masses under their mutual Newtonian gravitational forces, is the main problem of the classical Celestial Mechanics. Only the 2-body problem is completely solved, and for $n>2$ there are only few partial results on the dynamics of the $n$-body problem.

In $\mathbb{R}^{2}$ the equations of motion of the $n$-body problem are

$$
\ddot{x}_{i}=\sum_{j=1, j \neq i}^{n} \frac{m_{j}\left(x_{j}-x_{i}\right)}{r_{i j}^{3}}, \text { for } i=1, \ldots, n .
$$

where $x_{i} \in \mathbb{R}^{2}$ are the position vectors of the bodies, $r_{i j}=\left|x_{i}-x_{j}\right|$ are their mutual distances, and $m_{i}$ are their masses. Here the unit of time is taken in order that the Newtonian gravitational constant be equal to one.

The configuration of the system formed by the $n$ bodies is denoted by the vector $x=\left(x_{1}, \ldots, x_{n}\right) \in \mathbb{R}^{2 n}$. The differential equations of motion are well-defined when $r_{i j} \neq 0$ for $i \neq j$, i.e. when there is no collisions between the masses.

The dimension of the smallest affine subspace of $\mathbb{R}^{2}$ which contains all of the points $x_{i}$ is called the dimension $\delta(x)$ of the configuration $x$. Then the configurations with $\delta(x)=1,2$ are called collinear and planar, respectively.

We define the total mass and the center of mass of the $n$ bodies as

$$
M=m_{1}+\ldots+m_{n}, \quad c=\frac{1}{M}\left(m_{1} x_{1}+\cdots+m_{n} x_{n}\right),
$$

2010 Mathematics Subject Classification. Primary 70F07. Secondary 70F15.

Key words and phrases. convex central configuration, four-body problem, Hjelmslev quadrilateral. The second author is partially supported by MINECO grants MTM2013-40998-P and MTM201677278-P (FEDER) and the AGAUR grant 2014 SGR568. 
respectively. A configuration $x$ is central when the acceleration vectors of the bodies satisfy

$$
\sum_{\substack{j=1 \\ j \neq i}}^{n} \frac{m_{j}\left(x_{j}-x_{i}\right)}{r_{i j}^{3}}=\lambda\left(x_{i}-c\right), \quad \text { for } \quad i=1, \ldots, n
$$

where $\lambda$ is a constant.

We say that two central configurations are equivalent if there is a rotation of $S O(2)$ with respect to the center of mass and a homothecy which send one into the other. This relation is of equivalency, therefore in what follows when we talk on central configurations we are talking on classes of equivalency of central configurations.

The investigation of the central configurations begun at the second part of the 18th century. Early results on the central configurations can be found in the books by Wintner [43] and Hagihara [21]. Nowadays, there is an extensive literature on this subject, see among others Abouy and Chenciner [3], Albouy and Kaloshin [6], Hampton and Moeckel [23], Llibre [27], Moeckel [31], Palmore [35], Saari [39], Schmidt [40], Xia [44], ...

Central configurations are important for several reasons. First, they allow to obtain the homographic solutions of the $n$-body problem, which are the unique explicit solutions in function of the time known until now of that problem, for such solutions the ratios of the mutual distances between the bodies remain constant. Second, in the total collisions (i.e. all the bodies collide) or in the total parabolic escape to infinity (i.e. all the bodies escape to infinity with zero radial velocity) the configuration of the $n$ bodies is asymptotic to a central configuration, for more details see Dziobek [16] and [39]. Third, if we fix the first integrals of the total energy $h$ and the angular momentum $c$ of the $n$-body problem, then some of the bifurcation points $(h, c)$ for the topology of the level sets with energy $h$ and angular momentum $c$ depend on with the central configurations, for more details see Meyer [33] and Smale [42].

For a fixed mass vector $m=\left(m_{1}, \ldots, m_{n}\right)$ and a fixed ordering of the bodies along the line, Moulton [34] in 1910 proved, up to translation and scaling, that there exists a unique collinear central configuration. This result for the threebody problem was already obtained by Euler [18] in 1767. Also for the three-body problem Lagrange [24] proved that for the unique planar central configuration $x$ with $\delta(x)=2$ is the equilateral triangle, i.e. the three bodies are at the vertices of an equilateral triangle.

Here we want to improve the knowledge of the planar central configurations of the four-body problem. First we provide a brief summary of the results on the central configurations of the four-body problem. A numerical study on the classes of central configurations for the four-body problem with arbitrary masses was done by Simó in [41]. In 2006, Hampton and Moeckel [23] provided a computer assisted proof showing that the four-body problem has finitely many classes of central configurations with any given positive masses. Later on, this result was proved analytically by Albouy and Kaloshin [6]. 
Llibre in [26] found all the planar central configurations of the four-body problem with equal masses by studying the intersection points of two planar curves and assuming that such central configurations have an axis of symmetry. A complete analytic proof of this result was given later on by Albouy in $[1,2]$, showing that there are exactly four equivalent classes of central of these central configurations.

Pedersen [36], Barros and Leandro [10, 11], Gannaway [20] and Arenstorf [9] obtained numerically and analytically the classes of central configurations of the four-body problem when one of the four masses is sufficiently small.

A central configuration of the four-body problem having an axis of symmetry passing through two non-adjacent bodies is called kite. The non-collinear classes of kite central configurations with three equal masses where classified by Bernat et al. in [12], see also Leandro [25].

A planar configuration of the four-body problem is convex if none of the bodies is located in the interior of the triangle formed by the others, otherwise it is concave. MacMillan and Bartky [30] shown that for any assigned order of arbitrary four masses there is a convex planar central configuration of the four-body problem where the masses at the vertices of the quadrilateral are located in the given order. Later on Xia [45] gave an easier proof of this result.

In 2010, Piña and Lonngi [38] applied a new numerical algorithm to construct general four-body central configurations and described new properties of symmetric and non-symmetric central configurations.

Long and Sun [29] proved that any convex central configuration with masses $m_{1}=m_{2}<m_{3}=m_{4}$ such that the equal masses are located at opposite vertices of a quadrilateral and the diagonal corresponding to the mass $m_{1}$ is larger than or equal to the one corresponding to the mass $m_{3}$, has an axis of symmetry and the quadrilateral is a rhombus. Pérez-Chavela and Santoprete [37] extended this result to the case where two of the masses are equal and at most, only one of the remaining mass is larger than the equal masses. Furthermore, they shown that there exists a unique convex central configuration when the opposite masses are equal and it is a rhombus. Albouy et. al. [5] later on proved that a convex central configuration is symmetric with respect to one diagonal if and only if the masses of the two particles on the other diagonal are equal.

Álvarez-Ramírez and Llibre [7] characterized the convex and concave central configurations with an axis of symmetry of the four-body problem when the masses satisfy that $m_{1}=m_{2} \neq m_{3}=m_{4}$. On the other hand, Érdi and Czirják [17] derived a complete solution in a symmetric case of the planar four-body central configurations, when two bodies are on an axis of symmetry, and the other two bodies have equal masses and are situated symmetrically with respect to the axis of symmetry.

Albouy and $\mathrm{Fu}$ in [4] (see also [30, 37]) conjectured: For the planar four-body problem there is a unique convex central configuration for each ordering of the masses in the boundary of the convex hull of their positions. A particular case was considered before by MacMillan and Bartky [30], they proved that there is a unique isosceles trapezoid central configuration of the four-body when two pairs of 

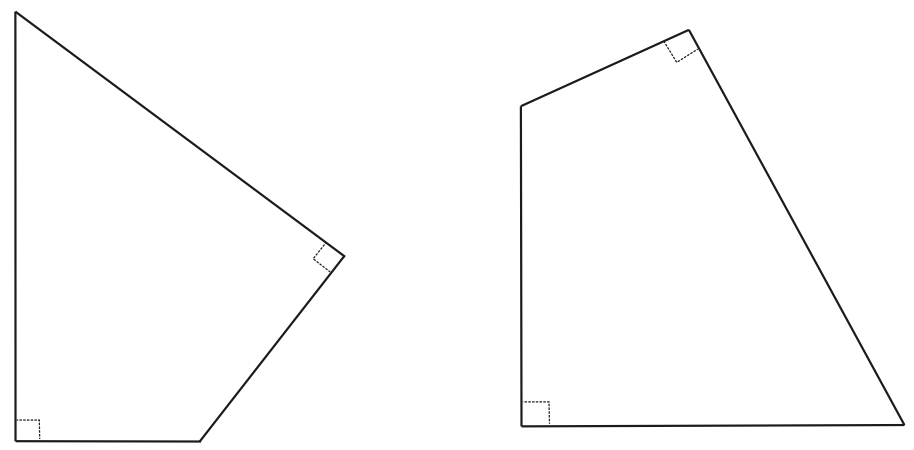

Figure 1. Hjelmslev quadrilateral.

equal masses are located at adjacent vertices. Later on Xie in [46] reproved this result.

There is also the following subconjecture: For the planar four-body problem there is a unique convex central configuration having two pairs of equal masses located at the adjacent vertices of the configuration and it is an isosceles trapezoid.

Using these previous results on the symmetries Corbera and Llibre [13] gave a complete description of the families of central configurations with two pairs of equals masses and two equal masses sufficiently small, proving for these masses the convex conjecture and the subconjecture. Recently Fernandes et al. [19] have proved the subconjecture for arbitrary masses.

The central configurations when the four masses are on a circle have been classified by Cors and Roberts [15], and also by Álvarez-Ramírez et al. [8]. A trapezoid is a convex quadrilateral with at least one pair of parallel sides. Corbera et al. [14] have classified all the trapezoids which are central configurations.

A Hjelmslev quadrilateral is a quadrilateral with two right angles at opposite vertices, see for more details about these kind of quadrilaterals the book [22], see Figure 1.

Since a central configuration is invariant under a homothecy and a rotation around its center of mass, without loss of generality we can assume that

$$
x_{1}=(0,0), \quad x_{2}=(1,0), \quad x_{3}=(a, b), \quad x_{4}=(0, c),
$$

where $a, b$ and $c$ are non-negative. Let $m_{k}>0$ be the mass of the particle located at $x_{k}$ for $k=1,2,3,4$.

The next lemma states that $c$ can be expressed in terms of $a$ and $b$ parameters. It is proved in Section 3.

Lemma 1. If the configuration of four masses is a Hjelmslev quadrilateral with vertices $x_{1}, x_{2}, x_{3}$ and $x_{4}$, as is shown in Figure 2, then

$$
c=\frac{a^{2}-a+b^{2}}{b} \text {. }
$$



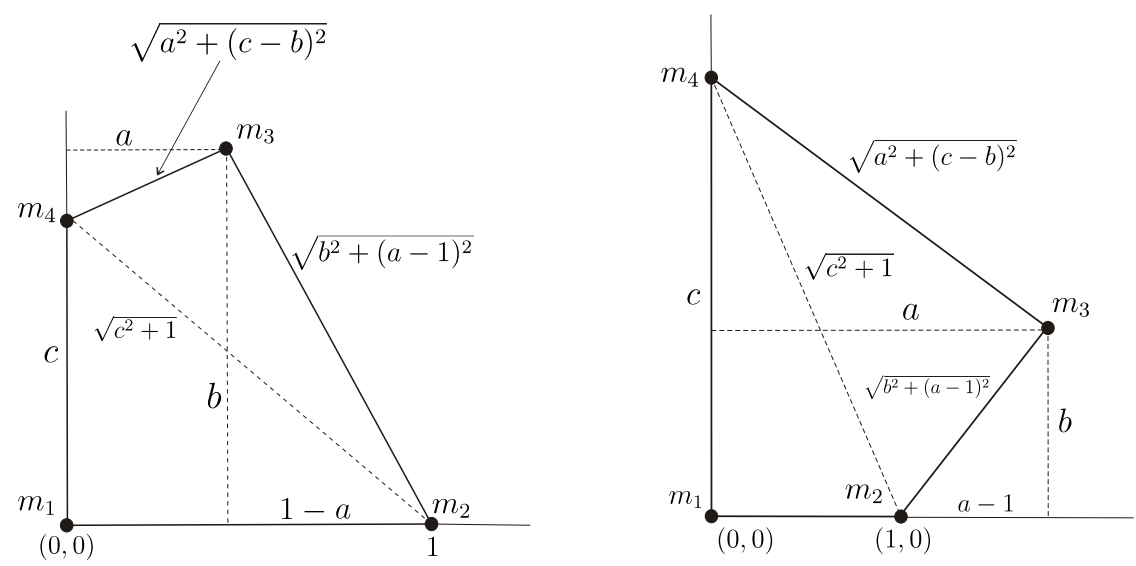

Figure 2. Coordinates of the four-body configuration forming a Hjelmslev quadrilateral for $a<1$ and $a>1$, respectively

We now give a result that allows to characterize all planar four-body central configurations where the four bodies are at the vertices of a Hjelmslev quadrilateral.

Theorem 2. We take positive masses for the four-body problem and the unit of mass equal to $m_{1}$. Then the points $(a, b)$ of the set of all Hjelmslev quadrilateral central configurations of the four-body problem described by (2) and (3) is formed by the open arc

$$
\Sigma=\left\{(a, b) \in\left(\frac{1}{2}, \frac{3}{2}\right) \times\left(\frac{\sqrt{3}}{2}, 1\right]: b=\sqrt{1-(a-1)^{2}}\right\} .
$$

(a) When the coordinates $(a, b)$ of the Hjelmslev quadrilateral central configurations tend to the boundary point $(1 / 2, \sqrt{3} / 2)$ of $\Sigma$ the Hjelmslev quadrilateral central configurations tend to the equilateral triangle central configuration of the three-body problem formed by the masses $m_{1}=1, m_{2}=$ $4(19+21 \sqrt{3}) / 37$ and $m_{3}=1$, the position of the remainder four body tends to $(0,1 / \sqrt{3})$ and its mass $m_{4} \rightarrow 0$.

(b) When the coordinates $(a, b)$ of the Hjelmslev quadrilateral central configurations tend to the boundary point $(3 / 2, \sqrt{3} / 2)$ of $\Sigma$ the Hjelmslev quadrilateral central configurations tend to the equilateral triangle central configuration of the three-body problem formed by the masses $m_{1}=1, m_{3}=$ $4(19+21 \sqrt{3}) / 37$ and $m_{4}=1$, the position of the remainder four body is $(0,1)$ and its mass $m_{2} \rightarrow 0$.

(c) The coordinates $(a, b)=(1,1) \in \Sigma$ correspond to the Hjelmslev quadrilateral central configuration given by the square with four equal masses at its vertices.

(d) Running the arc $\Sigma$ starting at $(1 / 2, \sqrt{3} / 2)$ and ending at $(3 / 2, \sqrt{3} / 2)$, the mass $m_{2}$ decreases monontically from $4(19+21 \sqrt{3}) / 37$ to zero, the mass $m_{3}$ remains constant equal to one, and the mass $m_{4}$ increases monontically from zero to $4(19+21 \sqrt{3}) / 37$. So every Hjelmslev quadrilateral central 
configuration is realized by a unique set of four masses, being two of the masses equal $\left(m_{1}=m_{3}=1\right)$, and the other two masses are different except in the square that the four masses are equal.

Theorem 2 is proved in Section 3. In Section 2, we recall the Dziobek's equations for the central configurations of the four-body problem.

\section{DZIOBEK'S EQUATIONS}

Let $x=\left(x_{1}, \ldots, x_{4}\right) \in\left(\mathbb{R}^{2}\right)^{4}$ be the configuration vector and we associate to each one the $4 \times 4$ matrix

$$
X=\left(\begin{array}{ccc}
1 & \cdots & 1 \\
x_{1} & \cdots & x_{4} \\
0 & \cdots & 0
\end{array}\right)
$$

We define the $3 \times 3$ matrix $X_{k}$ as the matrix obtained deleting from the matrix $X$ its $k$-th column and its last row. Then let $D_{k}=(-1)^{k+1} \operatorname{det}\left(X_{k}\right)$ be for $k=1, \ldots, 4$.

The equations for the central configurations (1) of the four-body problem were written by Dziobek [16] (see also equations (8) and (16) of Moeckel [32], or [21]) as the following 12 equations with 12 unknowns

$$
\begin{aligned}
& \frac{1}{r_{i j}^{3}}=c_{1}+c_{2} \frac{D_{i} D_{j}}{m_{i} m_{j}}, \\
& t_{i}-t_{j}=0,
\end{aligned}
$$

for $1 \leq i<j \leq 4$, with

$$
t_{i}=\sum_{j=1, j \neq i}^{4} D_{j} r_{i j}^{2} .
$$

In equations (4) the 12 unknowns are the 6 mutual distances $r_{i j}$, the 4 variables $D_{i}$, and the 2 constants $c_{k}$.

The first six Dziobek's equations (4) are

$$
\begin{aligned}
& m_{1} m_{2}\left(r_{12}^{-3}-c_{1}\right)=c_{2} D_{1} D_{2}, \\
& m_{1} m_{3}\left(r_{13}^{-3}-c_{1}\right)=c_{2} D_{1} D_{3}, \\
& m_{2} m_{3}\left(r_{23}^{-3}-c_{1}\right)=c_{2} D_{2} D_{3}, \\
& m_{1} m_{4}\left(r_{14}^{-3}-c_{1}\right)=c_{2} D_{1} D_{4}, \\
& m_{2} m_{4}\left(r_{24}^{-3}-c_{1}\right)=c_{2} D_{2} D_{4}, \\
& m_{3} m_{4}\left(r_{34}^{-3}-c_{1}\right)=c_{2} D_{3} D_{4}
\end{aligned}
$$

Multiplying the above equations by row in order that the product of the righthand side be simply $c_{2}^{2} D_{1} D_{2} D_{3} D_{4}$, and since the masses are positive we get the so called Dziobeck relation

$$
\left(r_{12}^{-3}-c_{1}\right)\left(r_{34}^{-3}-c_{1}\right)=\left(r_{13}^{-3}-c_{1}\right)\left(r_{24}^{-3}-c_{1}\right)=\left(r_{14}^{-3}-c_{1}\right)\left(r_{23}^{-3}-c_{1}\right),
$$


which holds for every planar central configuration of the 4-body problem. Solving with respect to $c_{1}$ any two of these equations we have

(7)

$$
c_{1}=\frac{r_{12}^{-3} r_{34}^{-3}-r_{13}^{-3} r_{24}^{-3}}{r_{12}^{-3}+r_{34}^{-3}-r_{13}^{-3}-r_{24}^{-3}}=\frac{r_{13}^{-3} r_{24}^{-3}-r_{14}^{-3} r_{23}^{-3}}{r_{13}^{-3}+r_{24}^{-3}-r_{14}^{-3}-r_{23}^{-3}}=\frac{r_{14}^{-3} r_{23}^{-3}-r_{12}^{-3} r_{34}^{-3}}{r_{14}^{-3}+r_{23}^{-3}-r_{12}^{-3}-r_{34}^{-3}} \text {. }
$$

If we set

$$
\begin{array}{ll}
s_{1}=r_{12}^{-3}+r_{34}^{-3}, & p_{1}=r_{12}^{-3} r_{34}^{-3}, \\
s_{2}=r_{13}^{-3}+r_{24}^{-3}, & p_{2}=r_{13}^{-3} r_{24}^{-3}, \\
s_{3}=r_{14}^{-3}+r_{23}^{-3}, & p_{3}=r_{14}^{-3} r_{23}^{-3},
\end{array}
$$

then equation (7) can be written as

$$
c_{1}=\frac{p_{1}-p_{2}}{s_{1}-s_{2}}=\frac{p_{2}-p_{3}}{s_{2}-s_{3}}=\frac{p_{3}-p_{1}}{s_{3}-s_{1}}
$$

which means that $\left(s_{1}, p_{1}\right),\left(s_{2}, p_{2}\right),\left(s_{3}, p_{3}\right)$ viewed as points in $\left(\mathbb{R}^{+}\right)^{2}$, must lie on the same line with slope $c_{1}$. This in turn, is equivalent to

$$
\left|\begin{array}{ccc}
1 & 1 & 1 \\
s_{1} & s_{2} & s_{3} \\
p_{1} & p_{2} & p_{3}
\end{array}\right|=0
$$

which allows to write Dziobeck relation (6) as the equation

(9) $D=\left(r_{13}^{3}-r_{12}^{3}\right)\left(r_{23}^{3}-r_{34}^{3}\right)\left(r_{24}^{3}-r_{14}^{3}\right)-\left(r_{12}^{3}-r_{14}^{3}\right)\left(r_{24}^{3}-r_{34}^{3}\right)\left(r_{13}^{3}-r_{23}^{3}\right)=0$,

that we call the Dziobeck equation $D=0$. Of course, the equation $D=0$ is satisfied for every planar central configuration of the four-body problem.

\section{Proof of Theorem 2}

Firstly we prove the Lemma 1.

Proof. Since the configuration is a Hjelmslev quadrilateral, using Figure 2 we have that $c^{2}+1=b^{2}+(a-1)^{2}+a^{2}+(c-b)^{2}$. Then a straightforward computation shows that $2 b^{2}+2 a^{2}-2 a-2 b c=0$. So, we have

$$
c=\frac{a^{2}-a+b^{2}}{b}
$$


From (2) and (3) we have

$$
\begin{aligned}
r_{12} & =1, & r_{13} & =\sqrt{a^{2}+b^{2}}, \\
r_{14} & =\frac{(a-1) a+b^{2}}{b}, & r_{23} & =\sqrt{(a-1)^{2}+b^{2}}, \\
r_{24} & =\sqrt{\frac{\left((a-1) a+b^{2}\right)^{2}}{b^{2}}+1,} & r_{34} & =\frac{a}{b} \sqrt{(a-1)^{2}+b^{2}}, \\
D_{1} & =\frac{a^{3}-2 a^{2}+a b^{2}+a}{b}, & D_{2} & =\frac{-a^{3}+a^{2}-a b^{2}}{b}, \\
D_{3} & =\frac{a^{2}-a+b^{2}}{b}, & D_{4} & =-b .
\end{aligned}
$$

By substituting these expression into last six Dziobek's equations (5), it is easy to see that are identically zero for a Hjelmslev quadrilateral configuration.

The following three equations

$$
\frac{m_{2}\left(r_{23}^{-3}-c_{1}\right)}{m_{1}\left(r_{13}^{-3}-c_{1}\right)}=\frac{D_{2}}{D_{1}}, \quad \frac{m_{3}\left(r_{23}^{-3}-c_{1}\right)}{m_{1}\left(r_{12}^{-3}-c_{1}\right)}=\frac{D_{3}}{D_{1}}, \quad \frac{m_{4}\left(r_{24}^{-3}-c_{1}\right)}{m_{1}\left(r_{12}^{-3}-c_{1}\right)}=\frac{D_{4}}{D_{1}}
$$

are obtained dividing the third equation of (5) by the second one, dividing the third equation of (5) by the first one, and dividing the fifth equation of (5)by the first one, respectively.

Taking the value of the mass $m_{1}$ as the unit of mass and using (7), we obtain that the three equations of (11) become

$$
m_{2}=\frac{D_{2} r_{23}^{3} r_{24}^{3}\left(r_{13}^{3}-r_{14}^{3}\right)}{D_{1} r_{13}^{3} r_{14}^{3}\left(r_{23}^{3}-r_{24}^{3}\right)}, m_{3}=\frac{D_{3} r_{23}^{3} r_{34}^{3}\left(r_{12}^{3}-r_{14}^{3}\right)}{D_{1} r_{12}^{3} r_{14}^{3}\left(r_{23}^{3}-r_{34}^{3}\right)}, m_{4}=\frac{D_{4} r_{24}^{3} r_{34}^{3}\left(r_{12}^{3}-r_{13}^{3}\right)}{D_{1} r_{12}^{3} r_{13}^{3}\left(r_{24}^{3}-r_{34}^{3}\right)} \text {. }
$$

Substituting these values of $m_{2}, m_{3}$ and $m_{4}$ into the first six Dziobek's equations (4), and taking only the numerators of these six equations because the denominators do not vanish, we obtain

$$
\begin{aligned}
e_{1}= & D_{2}\left(c_{2} D_{1}^{2} r_{12}^{3} r_{13}^{3} r_{14}^{3}\left(r_{24}^{3}-r_{23}^{3}\right)-r_{23}^{3} r_{24}^{3}\left(c_{1} r_{12}^{3}-1\right)\left(r_{13}^{3}-r_{14}^{3}\right)\right), \\
e_{2}= & D_{3}\left(c_{2} D_{1}^{2} r_{12}^{3} r_{13}^{3} r_{14}^{3}\left(r_{34}^{3}-r_{23}^{3}\right)-r_{23}^{3} r_{34}^{3}\left(c_{1} r_{13}^{3}-1\right)\left(r_{12}^{3}-r_{14}^{3}\right)\right), \\
e_{3}= & D_{2} D_{3}\left(r_{23}^{3} r_{24}^{3} r_{34}^{3}\left(c_{1} r_{23}^{3}-1\right)\left(r_{12}^{3}-r_{14}^{3}\right)\left(r_{14}^{3}-r_{13}^{3}\right)\right. \\
& \left.-c_{2} D_{1}^{2} r_{12}^{3} r_{13}^{3} r_{14}^{6}\left(r_{23}^{3}-r_{24}^{3}\right)\left(r_{23}^{3}-r_{34}^{3}\right)\right) \\
e_{4}= & D_{4}\left(c_{2} D_{1}^{2} r_{12}^{3} r_{13}^{3} r_{14}^{3}\left(r_{34}^{3}-r_{24}^{3}\right)-r_{24}^{3} r_{34}^{3}\left(c_{1} r_{14}^{3}-1\right)\left(r_{12}^{3}-r_{13}^{3}\right)\right), \\
e_{5}= & D_{2} D_{4}\left(-r_{23}^{3} r_{24}^{3} r_{34}^{3}\left(c_{1} r_{24}^{3}-1\right)\left(r_{12}^{3}-r_{13}^{3}\right)\left(r_{13}^{3}-r_{14}^{3}\right)\right. \\
& \left.-c_{2} D_{1}^{2} r_{12}^{3} r_{13}^{6} r_{14}^{3}\left(r_{23}^{3}-r_{24}^{3}\right)\left(r_{24}^{3}-r_{34}^{3}\right)\right) \\
e_{6}= & D_{3} D_{4}\left(c_{2} D_{1}^{2} r_{12}^{6} r_{13}^{3} r_{14}^{3}\left(r_{23}^{3}-r_{34}^{3}\right)\left(r_{34}^{3}-r_{24}^{3}\right)\right. \\
& \left.-r_{23}^{3} r_{24}^{3} r_{34}^{3}\left(c_{1} r_{34}^{3}-1\right)\left(r_{12}^{3}-r_{13}^{3}\right)\left(r_{12}^{3}-r_{14}^{3}\right)\right) .
\end{aligned}
$$

Now, we eliminate the factors $D_{\ell}$ from the equations (13), and they are non-zero. After we solve the first two equations with respect $c_{1}$ and $c_{2}$, and we substituted $c_{1}$ and $c_{2}$ in the last four equations, and then the system (13) is reduced to four 
equations, one of which is identically zero,

(14)

$$
\begin{aligned}
& e_{3}=\frac{D}{d} r_{23}^{6} r_{24}^{3} r_{34}^{3}\left(r_{14}-r_{12}\right)\left(r_{12}^{2}+r_{12} r_{14}+r_{14}^{2}\right)\left(r_{14}-r_{13}\right)\left(r_{13}^{2}+r_{13} r_{14}+r_{14}^{2}\right), \\
& e_{4}=0 \\
& e_{5}=\frac{D}{d} r_{23}^{3} r_{24}^{6} r_{34}^{3}\left(r_{13}-r_{12}\right)\left(r_{12}^{2}+r_{12} r_{13}+r_{13}^{2}\right)\left(r_{13}-r_{14}\right)\left(r_{13}^{2}+r_{13} r_{14}+r_{14}^{2}\right), \\
& e_{6}=\frac{D}{d} r_{23}^{3} r_{24}^{3} r_{34}^{6}\left(r_{13}-r_{12}\right)\left(r_{12}^{2}+r_{12} r_{13}+r_{13}^{2}\right)\left(r_{12}-r_{14}\right)\left(r_{12}^{2}+r_{12} r_{14}+r_{14}^{2}\right),
\end{aligned}
$$

where $D=0$ is the Dziobek equation (9), and

$$
d=r_{12}^{3}\left(r_{13}^{3} r_{23}^{3}\left(r_{24}^{3}-r_{34}^{3}\right)+r_{14}^{3} r_{24}^{3}\left(r_{34}^{3}-r_{23}^{3}\right)\right)+r_{13}^{3} r_{14}^{3} r_{34}^{3}\left(r_{23}^{3}-r_{24}^{3}\right)
$$

is the denominator which comes from the denominator of $c_{1}$ and $c_{2}$.

In summary, the Hjelmslev quadrilateral central configurations must satisfy the three equations $\left(e_{3}, e_{5}, e_{6}\right)=(0,0,0)$ of $(14)$.

Substituting (10) into $e_{3}=0, e_{5}=0$ and $e_{6}=0$, we get that these three equations are satisfied if and only if the next three equations have solutions

$$
\begin{aligned}
& E_{3}=D\left(1-\frac{(a-1) a+b^{2}}{b}\right)\left(\frac{(a-1) a+b^{2}}{b}-\sqrt{a^{2}+b^{2}}\right)=0, \\
& E_{5}=D\left(1-\sqrt{a^{2}+b^{2}}\right)\left(\frac{(a-1) a+b^{2}}{b}-\sqrt{a^{2}+b^{2}}\right)=0, \\
& E_{6}=D\left(1-\frac{(a-1) a+b^{2}}{b}\right)\left(1-\sqrt{a^{2}+b^{2}}\right)=0,
\end{aligned}
$$

respectively, where again $D=0$ is the Dziobek equation (9). Hence the unique solutions $(a, b)$ satisfying system $(15)$ are the ones satisfying $D=0$.

To compute the values of $a$ and $b$ for $D=0$, we used Mathematica to obtain a graphical solution as shown in Figure 3. Then, the set of solutions of system (15) is formed by two open upper half-cercles given by

$$
\Omega=\left\{(a, b) \text { : either } b=\sqrt{\frac{1}{4}-\left(a-\frac{1}{2}\right)^{2}}, \text { or } b=\sqrt{1-(a-1)^{2}}\right\} .
$$

It only remains to find values for $(a, b) \in \Omega$ for which the bodies in the Hjelmslev quadrilateral central configurations have positive masses.

Next, by assuming that $(a, b) \in \Omega$ and taking into account (12), from Fig. 3 we have numerical evidence that $m_{2}, m_{3}$ and $m_{4}$ are positive only for $b=$ $\sqrt{1-(a-1)^{2}}$ with $a \in(1 / 2,3 / 2)$. Furthermore, the masses are positive for $(a, b) \in(1,2 /, 3 / 2) \times\left(\frac{\sqrt{3}}{2}, 1\right]$ and we have the following conclusion:

(i) $m_{4}=-1$ on the arc of $\Omega$ contained into the circle of radius $1 / 2$;

(ii) $m_{3}=1$ on the arc of $\Omega$ contained into the circle of radius 1 ;

(iii) the mass $m_{2}$ only is positive on the subarc of $\Omega$ contained into the circle of radius 1 when $a \in(1,3 / 2)$, in this subarc the mass $m_{2}$ decreases from 


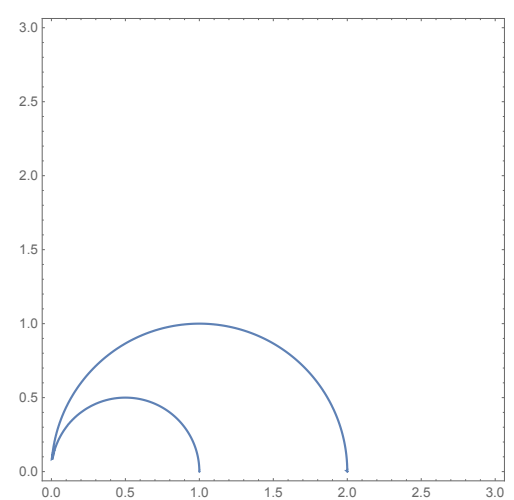

Figure 3. The graphic of the curve $D=0$ in the square $(a, b) \in[0,3]^{2}$.

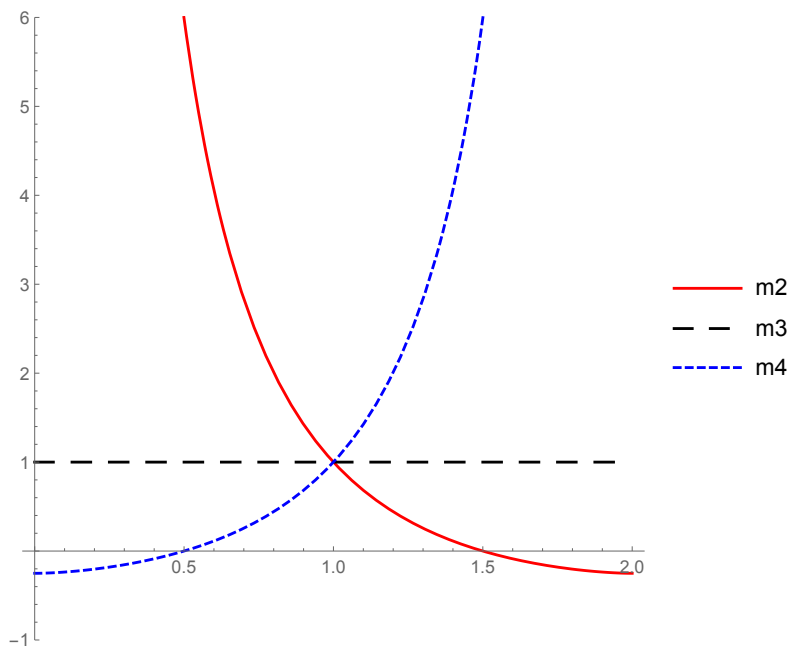

Figure 4. The curve $m_{1}, m_{2}$ and $m_{3}$ for $b=\sqrt{1-(a-1)^{2}}$ with $a \in[0,2]$.

1 to 0 , and in the point $(1 / 2, \sqrt{3} / 2)$ takes the value $4(19+21 \sqrt{3}) / 37$, see Figure 4;

(iv) the mass $m_{4}$ only is positive on the subarc of $\Omega$ contained into the circle of radius 1 when $a \in(1 / 2,2)$, in this subarc the mass $m_{4}$ increases from 0 to $+\infty$, and in the point $(3 / 2, \sqrt{3} / 2)$ takes the value $4(19+21 \sqrt{3}) / 37$, see Figure 4.

From (i)-(iv) it follows the statements of Theorem 2.

\section{REFERENCES}

[1] A. Albouy, Symétrie des configurations centrales de quarte corps, C. R. Acad. Sci. Paris 320 (1995), 217-220.

[2] A. Albouy, Recherches sur le problème des $n$ corps, Notes Scientifiques et Techniques du Bureau des Longitudes, Paris (1997), 78 pp. 
[3] A. Albouy And A. Chenciner, Le problème des $n$ corps et les distances mutuelles, Invent. math. 131 (1998), 151-184.

[4] A. Albouy And Y. Fu, Euler configurations and quasi polynomial systems, Regul. Chaotic Dyn. 12 (2007), 39-55.

[5] A. Albouy, Y. Fu And S. Sun, Symmetry of planar four-body convex central configurations, Proc. R. Soc. Lond. Ser. A 464 (2008), 1355-1365.

[6] A. Albouy and V. Kaloshin, Finiteness of central configurations of five bodies in the plane, Annals of Math. (2) 176 (2012), 535-588.

[7] M. Álvarez-Ramírez And J. Llibre, The symmetric central configurations of the 4-body problem with masses $m_{1}=m_{2} \neq m_{3}=m_{4}$, Appl. Math. Comput. 219 (2013), no. 11, 5996-6001.

[8] M. Álvarez-Ramírez, A. A. Santos And C. Vidal, On co-circular central configurations in the four and five body-problems for homogeneous force law, J. Dynam. Differential Equations 25 (2013), no. 2, 269-290.

[9] R.F. Arenstorf, Central configurations of four bodies with one inferior mass, Cel. Mech. 28 (1982), 9-15.

[10] J.F. BARros AND E.S.G. LEANDRo, The set of degenerate central configurations in the planar restricted four-body problem, SIAM Journal on Mathematical Analysis 43 (2011), 634-661.

[11] J.F. BARRos AND E.S.G. LEANDRO, Bifurcations and enumeration of classes of relative equilibria in the planar restricted four-body problem, SIAM Journal on Mathematical Analysis 46 (2014), 1185-1203.

[12] J. Bernat, J. Llibre and E. Perez-Chavela, On the planar central configurations of the 4-body problem with three equal masses, Dyn. Contin. Discrete Impuls. Syst. Ser. A Math. Anal. 16 (2009), 1-13.

[13] M. Corbera AND J. Llibre, Central configurations of the 4-body problem with masses $m_{1}=$ $m_{2}>m_{3}=m_{4}=m>0$ and $m$ small, Appl. Math. Comput. 246 (2014), 121-147.

[14] M. Corbera, J.M. Cors, J. Llibre and E. Perez-Chavela, Trapezoid central configurations, arXiv:1712.07155 [math.DS], 2017.

[15] J.M. Cors And G.E. RoberTs, Four-body co-circular central configurations, Nonlinearity 25 (2012), 343-370.

[16] O. Dzıовек, Ueber einen merkwürdigen Fall des Vielkörperproblems, Astron. Nach. 152 (1900), 32-46.

[17] B. ÉRDi AND Z. CzIRJÁK, Central configurations of four bodies with an axis of symmetry, Celestial Mech. Dynam. Astronom. 125 (2016), no. 1, 33-70.

[18] L. Euler, De moto rectilineo trium corporum se mutuo attahentium, Novi Comm. Acad. Sci. Imp. Petrop. 11 (1767), 144-151.

[19] A.C. Fernandes, J. Llibre And L.F. Mello, Convex central configurations of the 4-body problem with two pairs of equal masses, Arch. Rational Mech. Anal. 226 (2017), 303-320.

[20] J.R. Gannaway, Determination of all central configurations in the planar 4-body problem with one inferior mass, $\mathrm{Ph}$. D., Vanderbilt University, Nashville, USA, 1981.

[21] Y. Hagihara, Celestial Mechanics, vol. 1, chap. 3. The MIT Press, Cambridge, 1970.

[22] R. Hartshorne, Geometry: Euclid and Beyond, Springer, 2005, pp. 429-430.

[23] H. Hampton And R. Moeckel, Finiteness of relative equilibria of the four-body problem, Inventiones math. 163 (2006), 289-312.

[24] J.L. LAgRange, Essai sur le problème des trois corps, recueil des pièces qui ont remporté le prix de l'Académie Royale des Sciences de Paris, tome IX, 1772, reprinted in Ouvres, Vol. 6 (Gauthier-Villars, Paris, 1873), pp 229-324.

[25] E.S.G. LEANDro, Finiteness and bifurcation of some symmetrical classes of central configurations, Arch. Rational Mech. Anal. 167 (2003), 147-177.

[26] J. Llibre, Posiciones de equilibrio realtivo del problema de 4 cuerpos, Publicacions Matemàtiques UAB 3 (1976), 73-88.

[27] J. LliBRE, On the number of central configurations in the N-body problem, Cel. Mech. Dynam. Astronom. 50 (1991), 89-96.

[28] Y. Long, Admissible shapes of 4-body non-collinear relative equilibria, Adv. Nonlinear Stud. 3 (2003), no. 4, 495-509.

[29] Y. Long And S. Sun, Four-body central configurations with some equal masses, Arch. Rational Mech. Anal. 162 (2002), 24-44. 
[30] W.D. Macmillan and W. Bartky, Permanent Configurations in the Problem of Four Bodies, Trans. Amer. Math. Soc. 34 (1932), 838-875.

[31] R. Moeckel, On central configurations, Mah. Z. 205 (1990), 499-517.

[32] R. Moeckel, Generic finiteness for Dziobek configurations, Trans. Amer. Math. Soc. 353 (2001), 4673-4686.

[33] K.R. Meyer, Bifurcation of a central configuration, Cel. Mech. 40 (1987), 273-282.

[34] F.R. Moulton, The straight line solutions of $n$ bodies, Ann. of Math. 12 (1910), 1-17.

[35] J.I. Palmone, Classifying relative equilibria II, Bull. Amer. Math. Soc. 81 (1975), 71-73.

[36] P. Pedersen, Librationspunkte im restringierten Vierk" orperproblem, Danske Vid. Selsk. Math.-Fys. 21 (1944), 1-80.

[37] E. PÉrez-Chavela and M. Santoprete, Convex four-body central configurations with Some equal masses, Arch. Rational Mech. Anal. 185 (2007), 481-494.

[38] E. PiÑa AND P. Lonngi, Central configurations for the planar Newtonian four-body problem, Celest. Mech. Dyn. Astron. 108, 73-93 (2010).

[39] D. SAARI, On the role and properties of central configurations, Cel. Mech. 21 (1980), 9-20.

[40] D.S. Schmidt, Central configurations in $\mathbb{R}^{2}$ and $\mathbb{R}^{3}$, Contemporary Math. 81 (1980), 59-76.

[41] C. Simó, Relative equilibrium solutions in the four-body problem, Cel. Mech. 18 (1978), 165184 .

[42] S. Smale, Topology and mechanics II: The planar n-body problem, Inventiones math. 11 (1970), 45-64.

[43] A. Wintner, The Analytical Foundations of Celestial Mechanics, Princeton University Press, 1941

[44] Z. XIA, Central configurations with many small masses, J. Differential Equations 91 (1991), $168-179$.

[45] Z. XIA, Convex central configurations for the $n$-body problem, J. Differential Equations 200 (2004), 185-190.

[46] Z. XIE, Isosceles trapezoid central configurations of the Newtonian four-body problem, Proc. R. Soc. Edinb., Sect. A, Math. 142 (2012), 665-672.

1 Departamento de Matemáticas, UAM-Iztapalapa, San Rafael Atlixco 186, Col. ViCentina, 09340 Iztapalapa, México, City, México.

E-mail address: mar@xanum.uam.mx

${ }^{2}$ Departament de Matemàtiques, Universitat Autònoma de Barcelona, 08193 Bellaterra, Barcelona, Catalonia, Spain

E-mail address: jllibre@mat.uab.cat 